\title{
Transcatheter Mitral Valve-in-Valve Implantation in a 13-Year-Old Female with a Degenerated Bioprosthetic Mitral Valve: Case Report
}

\author{
Reda Abuelatta ${ }^{1}$ and Hesham Naeim ${ }^{1}$ \\ ${ }^{1}$ Madinah Cardiac Center
}

May 12, 2020

\begin{abstract}
Background: On June 05, 2017, the U.S. Food and Drug Administration approved the Edwards Lifesciences SAPIEN S3 Valve for transcatheter mitral valve replacement of degenerated bioprosthetic mitral valves. We present a case of the youngest patient (13 years old) to undergo this procedure. Case presentation: At 10 years old, this female patient underwent bioprosthetic mitral valve replacement. Three years later, she began experiencing shortness of breath NYHA class III. A transthoracic echocardiography showed a degenerated bioprosthetic mitral valve and severe mitral stenosis with a mean pressure gradient of $18 \mathrm{~mm} \mathrm{Hg}$. After deeming the patient, a high risk for surgery, the heart team opted to perform a transcatheter mitral valve-in-valve replacement. After the valve delivery system crossed the degenerated valve, a SAPIEN valve balloon was slowly inflated without rapid pacing to allow for fine positioning. Transoesophageal echocardiography evaluation revealed a mean gradient of $5 \mathrm{~mm} \mathrm{Hg}$. The patient discharged after 2 days. At her 30-month follow-up, she reported regular daily activity with no shortness of breath, and a follow-up transthoracic echocardiography showed normal function of the right ventricle, which also had reduced in size. Her systolic pulmonary artery pressure decreased to $33 \mathrm{~mm} \mathrm{Hg}$, the mean pressure gradient of the left ventricular outflow tract was $10 \mathrm{~mm} \mathrm{Hg}$, and the mitral valve mean pressure gradient was $6 \mathrm{~mm} \mathrm{Hg}$. Case discussion: Transcatheter mitral valve replacement is feasible in young female patients with a small body surface area. Safety and long-term outcomes need more research in this age group.
\end{abstract}

\section{Introduction}

Surgical mitral valve replacement has been the mainstay treatment for degenerated bioprosthesis. However, the transcatheter valve-in-valve procedure is increasingly recognized as an alternative treatment in selected patients. ${ }^{1}$ Moreover, percutaneous valve technology continues to evolve, making these valves available for use in a broader population. ${ }^{1}$ In 2017, the American Heart Association/American College of Cardiology updated its guidelines for management of patients with valvular heart disease, changing the word "reoperation" to "reintervention" to account for transcatheter valves. ${ }^{2}$ However, although bioprosthetic valves have shown good reliability in older patients, they degenerate more quickly in younger patients, presumably because of the increased contractility and valve stress among those with increased activity. ${ }^{3}$ We present a case involving a 13-year old female who received a transcatheter valve for degenerated mitral bioprosthesis with 30 months of follow up. To the best of our knowledge, this is the youngest patient to undergo this procedure.

\section{History}

A 10-year-old girl with known rheumatic heart disease presented with severe shortness of breath. She underwent a bioprosthetic mitral valve (MV) replacement (23-mm Perimount bovine pericardial leaflets sutured inside the stent) due to severe combined mitral stenosis and regurgitation. She did well for the next 2 years and then developed shortness of breath NYHA class III that lasted 12 months. 


\section{Case Presentation}

The child's weight was $38 \mathrm{~kg}$, her height was $1.48 \mathrm{~m}$, 32th percentile according to the Centers for Disease Control and Prevention (CDC) growth charts.. An examination revealed a diastolic rumbling murmur (grade IV/VI) with maximum intensity at the apex and propagation to the axilla. Transthoracic echocardiography showed a degenerated bioprosthetic MV with thickened, calcific, and restricted leaflets. The right ventricle (RV) was severely dilated with significantly depressed function, and the interventricular septum shifted substantially towards the left side (Videos 1 and 2). The aorta-MV angle was $63^{\circ}$ (Figure 1A), the left ventricular outflow tract (LVOT) diameter was $19 \mathrm{~mm}$, and the LVOT mean pressure gradient was $5 \mathrm{~mm}$ $\mathrm{Hg}$ with no septal bulge. Thus, LVOT obstruction was unlikely. Severe mitral stenosis was observed, with a mean pressure gradient of $18 \mathrm{~mm} \mathrm{Hg}$ (Figure 1B). There was mild tricuspid regurgitation, with an estimated systolic pulmonary artery pressure of $66 \mathrm{~mm} \mathrm{Hg}$ (Figure 1C). Transoesophageal echocardiography confirmed these findings (Figure 1D and Video 3), and 3D zoom of the MV from the left atrium and left ventricle showed a fusion of the three leaflets (Figure $1 \mathrm{E}$ and $1 \mathrm{~F}$ and Video 4). The heart team deemed her a high risk for surgery due to severe RV dilation, RV dysfunction, and severe pulmonary hypertension. The team thus recommended a transcatheter mitral valve replacement (TMVR).

The procedure was conducted in a standard catheterization laboratory under general anaesthesia and with transoesophageal echocardiography guidance. Right femoral arterial and venous accesses were established, and left venous access $6 \mathrm{~F}$ for the pacemaker lead was established as a backup. Transseptal access was difficult due to the heavily fibrotic and thick septum. A diathermy pen in cautery mode was applied to the hub of a BRK transseptal needle for 2 seconds, which facilitated septum crossing. A 5-F multipurpose catheter (Cook Medical, Bloomington, IN, USA) was advanced over a standard 0.035-inch guidewire using an Agilis catheter (St. Jude Medical, St. Paul, MN, USA). A Glidewire(r) (Terumo Medical Corporation, Somerset, NJ, USA) facilitated crossing of the mitral valve bioprosthesis (Figure 2A). A stiff, pre-shaped, 0.035-inch Confida Wire (Medtronic, Minneapolis, MN, USA) was positioned in the left ventricle apex. An Armada 35 peripheral 14-mm balloon (Abbott, Santa Clara, CA, USA) was used to dilate the atrial septum (Figure $2 \mathrm{~B}$ ), followed by dilatation of the stenosed valve using the same balloon (Figure 2C and 2D).

An Edwards E-sheath $14 \mathrm{~F}$ was introduced through the right femoral vein to the level of the hepatic veins. A 23-mm SAPIEN 3 valve was crossed to the left atrial cavity with the Edwards mark facing upside down to improve flexion via the macro knob. Unfortunately, the system loop inside the left atrium failed to cross. We attempted to establish apical left ventricular access to allow for system stability, unfortunately the access was established in the right ventricular apex due to the severe enlargement of the right ventricle. Confida wire was snared through the aortic valve (Figure 2E) to facilitate crossing the bioprosthesis.

After the valve delivery system crossed the degenerated valve, the SAPIEN valve balloon was inflated slowly without need for rapid pacing to enable positioning on $80 \%$ of the ventricular and $20 \%$ of the atrial valve stent (Figure 2F, Figure 3A, and Video 5). The balloon was inflated an additional $2 \mathrm{ml}$ for ventricular flaring of the SAPIEN valve. A multipurpose catheter facilitated removal of the Confida wire (Figure 3B). Transoesophageal echocardiography evaluation revealed a mean gradient of $5 \mathrm{~mm} \mathrm{Hg}$ with no valvular or paravalvular incompetence (Figure 3C). 3D imaging showed a well-opened valve (Figures 3D and 3E and Video6). The unintentional RV apical access was closed using an 8-mm Amplatzer Vascular Plug II (St. Jude Medical, St. Paul, MN, USA), as shown in Figure 3F and Video 7. The patient was discharged after 2 days.

At the patient's 30-month follow-up, her weight was $50 \mathrm{~kg}$, height was $1.5 \mathrm{~m}$, 70th percentile according to the CDC growth charts. She had regular daily activity with no shortness of breath. Transthoracic echocardiography revealed that the RV reduced in size and demonstrated normal functioning in comparison to preoperative images (Figure $4 \mathrm{~A}$ and $4 \mathrm{~B}$ and Video 8 ). Systolic pulmonary artery pressure reduced to 33 $\mathrm{mm} \mathrm{Hg}$ (Figure 4C), the LVOT mean pressure gradient at LVOT was $10 \mathrm{~mm} \mathrm{Hg}$ (Figure 4D), and the MV mean pressure gradient was $6 \mathrm{~mm} \mathrm{Hg}$ (Figure 4E).

\section{Discussion}


TMVR is a new hope for young patients with degenerated bioprosthetic mitral valves, as it postpones or eliminates the need for repeat surgeries, especially among women of childbearing age. However, TMVR is not a regular treatment option at this age and should be considered only in selected high-risk paediatric patients. Recent retrospective publications have shown excellent hemodynamic results for TMVR, with modest adverse events and short hospital stays. ${ }^{4}$ Transoesophageal echocardiography guides accurate prosthesis size, depth of insertion, and anchoring to avoid LVOT obstruction. The risk for LVOT obstruction increases in the presence of small baseline LVOT dimensions, basal septal bulge, or a large angle between the trajectory of the existing bioprosthesis and the aortic root. ${ }^{5}$ During TMVR, the pre-existing bioprosthetic valve leaflets can cover the outer frame of the new valve, thereby creating a potentially narrow "neo" LVOT. ${ }^{6}$

Accurate assessment of surgical heart valve anatomy is essential to optimize TMVR outcomes. Accurate sizing for the TMVR procedure requires the smallest true internal dimension of the surgical bioprosthesis. ${ }^{7}$ Because the true internal dimension is not routinely reported by manufacturers, a free ViV Mitral app was created by V.B. and UBQO Limited (London, United Kingdom). This app provides the true internal dimensions and recommended TMVR sizes for each bioprosthesis. ${ }^{8}$ Oversizing by $10 \%$ may be necessary for the mitral prosthesis, however, given reports of late migration. ${ }^{9}$

Careful serial follow-up of TMVR is essential to detect and reverse early valve thrombosis. ${ }^{10}$ The Valvein-Valve International Data (VIVID) registry includes 660 patients (mean age 73+-12 years) who have undergone TMVR procedures. The expected durability of the bioprosthetic valve averages 8.5 years since last cardiac surgery. ${ }^{11}$ However, it is known to degenerate earlier in younger patients. In this case, the bioprosthetic mitral valve degenerated after 3 years in a 13-year-old female patient. Many valves can be implanted percutaneously in young patients until they reach adulthood, at which time durable surgical mechanical valves can be implanted. Additional prospective, multi-centre, longitudinal research is essential to better understand the long-term risks and benefits of TMVR, especially in young patients.

\section{Conclusion}

This case study indicates that TMVR is feasible in young female patients who have small body surface areas. However, safety and long-term outcomes need more research in this age group.

\section{Conflict of interest}

No conflict of interest.

\section{Abbreviations}

Transcatheter mitral valve replacement (TMVR)

Mitral valve (MV)

Right ventricle (RV)

Left ventricular outflow tract (LVOT)

Valve-in-Valve International Data Registry (VIVID)

\section{References}

1. Mankad SV, Aldea GS, Ho NM, Mankad R, Pislaru S, Rodriguez LL, Whisenant B, et al. Transcatheter Mitral Valve Implantation in Degenerated Bioprosthetic Valves. J Am Soc Echocardiogr 2018;31:84559.

2. Nishimura RA, Otto CM, BonowRO, Carabello BA, Erwin JP, Fleisher LA, et al. 2017 AHA/ACC focused update of the 2014 AHA/ACC guideline for the management of patients with valvular heart disease: a report of the American College of Cardiology/American Heart Association task force on clinical practice guidelines. Circulation 2017;135: E1159-95.

3. Goldstone AB, Chiu P, Baiocchi M, Lingala B, Patrick WL, Fischbein MP, et al. Mechanical or biologic prostheses for aortic-valve and mitral-valve replacement. N Engl J Med 2017;377:1847-57. 
4. Petronio AS, Capranzano P, Barbato E, Piazza N, Baumbach A, Haude M, et al. Current status of transcatheter mitral valve therapy in Europe: results from an EAPCI survey (part II). Eurointervention 2017;12:1934-9.

5. Kwon DH, Smedira NG, Popovic ZB, Lytle BW, Setser RM, Thamilarasan M, et al. Steep left ventricle to aortic root angle and hypertrophic obstructive cardiomyopathy: study of a novel association using three-dimensional multimodality imaging. Heart 2009;95:1784-91.

6. Blanke P, Naoum C, Dvir D, Bapat V, Ong K, Muller D, et al. Predicting LVOT obstruction in transcatheter mitral valve implantation: concept of the neo-LVOT. JACC Cardiovasc Imaging 2017;10:4825.

7. Alia Noorani, Rizwan Attia and Vinayak Bapat. Valve-in-valve procedure: importance of the anatomy of surgical bioprostheses doi:10.1093/mmcts/mmu020 published online 2014.

8. V. Bapat, Valve-in-valve apps: why and how they were developed and how to use them EuroIntervention, 10 (Suppl U) (2014), pp. U44-U51

9. V. Bapat, F. Khaliel, L. Ihleberg Delayed migration of SAPIEN valve following a transcatheter mitral valve-in-valve implantation Catheter Cardiovasc Interv, 83 (2014), pp. E150-E154

10. Hudec V, Bena M, Artemiou P, Gasparovic I, Hulman M. Reversible thrombotic mitral valve stenosis after transcatheter mitral valve replacement (TMVR): is life-long anticoagulation therapy necessary? J Card Surg 2017;32:190-2.

11. Henrique Ribeiro, On behalf of VIVID registry investigators. https://valveinvalve.org/2018/10/16/mitral-viv/

\section{Figure legends}

Figure 1. A: Degenerated calcific bioprosthetic mitral valve, with aorta-mitral valve angle of 63deg. B: Mean pressure gradient of mitral valve is $18 \mathrm{~mm} \mathrm{Hg}$. C: Estimated systolic pulmonary artery pressure from the tricuspid regurgitation jet is $66 \mathrm{~mm} \mathrm{Hg}$. D: Transoesophageal echocardiography 2D shows degenerated bioprosthesis. E: 3D zoom from the left atrium (diastolic frame) shows mitral valve not opening. F: 3D zoom from the left ventricle shows thickened and calcified mitral valve.

Figure 2. A: Transseptal wire passing the degenerated mitral valve. B: Balloon dilation (14-mm) of interatrial septum. C: 3D balloon dilation of the bioprosthetic mitral valve. D: Balloon dilation of degenerated mosaic bioprosthetic mitral valve. E: Snaring of the wire from the left ventricle to the aorta. F: Deployment of the 23-mm Edwards SAPIEN 3 valve.

Figure 3. A: 3D deployment of 23-mm Edwards SAPIEN 3 valve. B: Fluoroscopy of deployed valve. C: Mean pressure gradient of valve-in-valve is $5 \mathrm{~mm} \mathrm{Hg}$. D: 2D colour image of deployed valve. E: 3D image of deployed valve in diastole showing valve opening well. F: Vascular plug closing the right ventricle apical puncture.

Figure 4. A: Apical, four-chamber view before mitral valve-in-valve with dilated right ventricle. B: Apical, 4-chamber view at 30-month follow-up showing smaller right ventricle. C: Estimated systolic pulmonary artery pressure at 30-month follow-up is $33 \mathrm{~mm} \mathrm{Hg}$. D: Mean pressure gradient at left ventricular outflow tract is $10 \mathrm{~mm} \mathrm{Hg}$, indicating no obstruction. E: Mean pressure gradient of mitral valve-in-valve at 30-month follow-up is $6 \mathrm{~mm} \mathrm{Hg}$.

\section{Video legends}

Video 1: Transthoracic echocardiography parasternal long axis view showing thickened calcific bioprosthetic mitral valve and dilated right ventricle with depressed function

Video 2: Transthoracic echocardiography apical 4 chamber view showing severely dilated right ventricle with severe dysfunction compressing the left ventricle

Video 3: Transoesophageal echocardiography mid-oesophageal view showing degenerated and severely stenotic bioprosthetic mitral valve 
Video 4: Transoesophageal echocardiography 3D surgical view showing degenerated bioprosthetic mitral valve with three fused leaflets

Video 5: Fluoroscopy showing 23-valve deployment

Video 6: Transoesophageal echocardiography 3D zoom showing well-opened valve

Video 7: Fluoroscopy showing device closure of unintentional right ventricle apical access

Video 8: Transthoracic echocardiography at 30-month follow-up showing reduced right ventricle and improved function

\section{Timeline}

January 2013

February 2015

April 2016

Follow up after 30 months
At 10 years old, a female patient with rheumatic heart disease presents with severe shortness 0 At 12 years old, patient exhibits shortness of breath (class II) that progresses to class III in th At 13-year-old, she was presented with severe SOB and admitted to the hospital.

A transthoracic echocardiography shows a degenerated bioprosthetic mitral valve and severely The heart team discussed the case and opted not to perform surgery. The team recommended The transcatheter mitral valve replacement was successfully completed. The patient's mean pr At the patient's 30-month post-procedure follow-up, she reported regular daily activity with no

\section{Hosted file}

Title page.docx available at https://authorea.com/users/320978/articles/450353-transcathetermitral-valve-in-valve-implantation-in-a-13-year-old-female-with-a-degeneratedbioprosthetic-mitral-valve-case-report
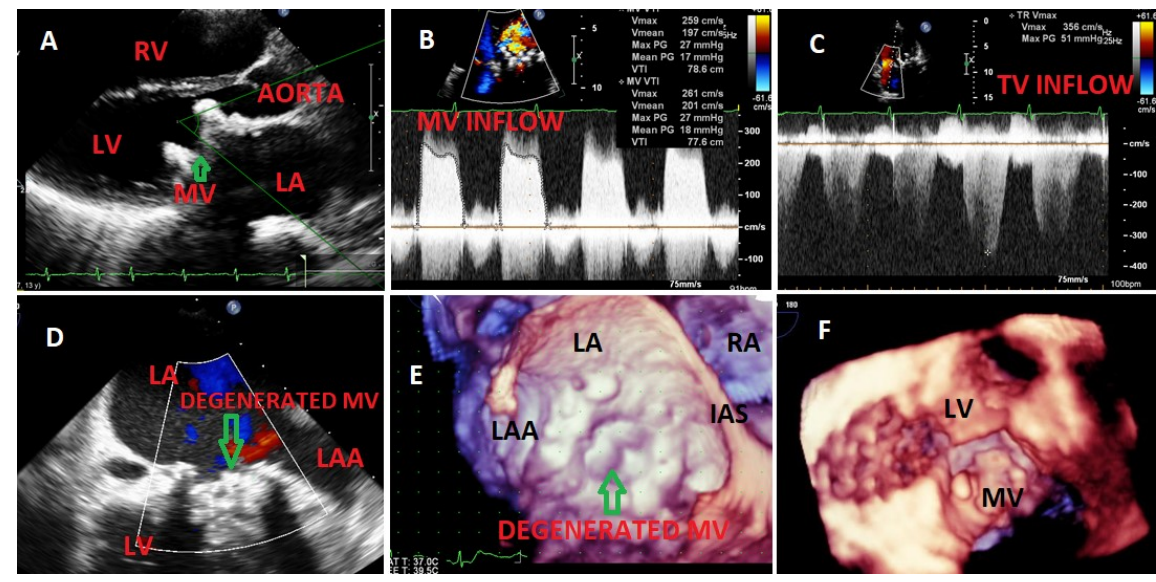

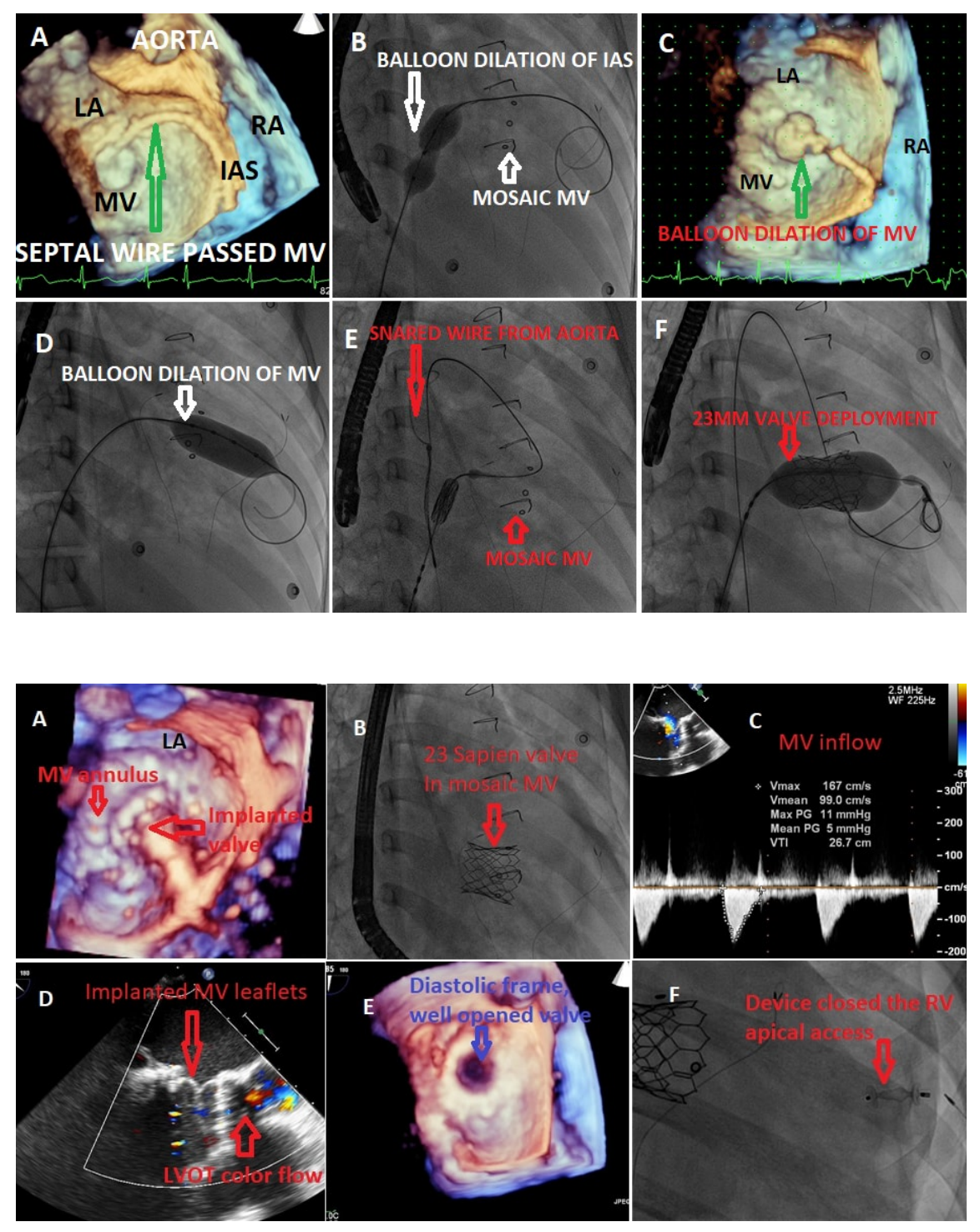


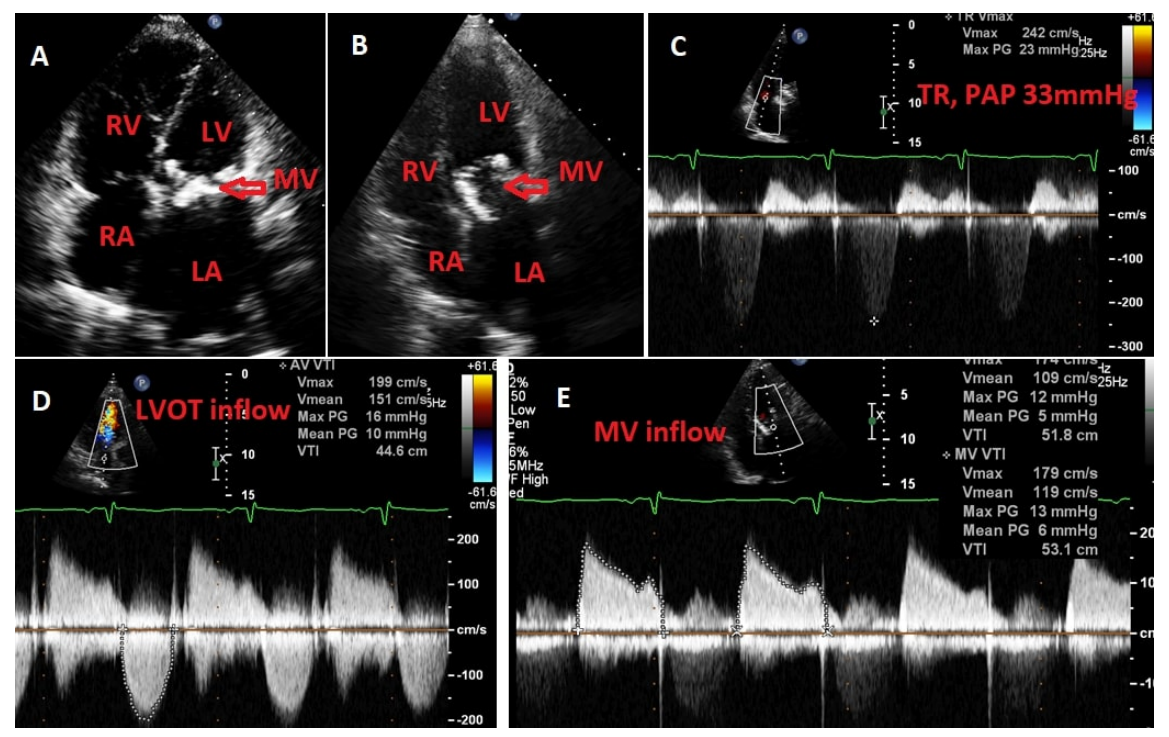

